\title{
Annotation
}

\section{Intraindividual Root-Length Correlations}

\author{
S. M. GARn, W. L. VAN Alstine, JR, and P. E. Cole
}

Center for Human Growth and Development, The University of Michigan, Ann Arbor Michigan 48109, USA

\section{J Dent Res 57(2): 270, February 1978}

Although crown diameters can be measured in both living individuals (through the medium of casts) as well as in skeletalized material and in isolated teeth, rather little attention has been given to root lengths and their interrelationships, especially in the course of in vivo studies.

We conducted a pilot investigation, therefore, measuring mandibular tooth root lengths on $45^{\circ}$ oblique head plates of 16 - and 17 -year old participants in a longitudinal study. Since year-to-year correspondences for root lengths of $\mathrm{C}-\mathrm{M}_{2}$ averaged 0.72 from tracings and 0.88 as repeatedly measured on head plates of 33 boys and girls, intertooth correlations were calculated from the direct micrometer caliper measurements for a larger series of 122. For the larger series ( 68 boys and 53 girls), and pooling sexspecific correlations, meaningful general trends emerged, as shown in the table. Briefly stated, adjacent teeth exhibited the highest root-length correlations ( $r=0.63)$; teeth once removed in the dental arch evidenced an average $r$ of 0.51 , while those twice removed (i.e. separated by 2 teeth) were characterized by a mean $r$ of 0.46 . The highest intertooth root length correlation was 0.67 for $P 1$ and $P 2$, and the lowest $(0.35)$ involved $\mathrm{C}$ and $\mathrm{M}_{2}$. These findings are consis. tent with a distance "field" of decreasing inten-

Received for publication October 25, 1977.

Accepted for publication January 27, 1978.

This study was supported in part by Grant DE.03610 from the National Institures of Health. sity particularly affecting the roots of adjacent teeth.

Within correlation limits set by sample size therefore (GARN, COLE, and GUIRE, $J$ Dent Res $56: 697,1977$ ), this study confirms the existence of intraindividual and intertooth similarities in root length that correspond surprisingly well to intraindividual crown-size resemblances previously reported by us (GARN, LEWIS and KEREWSKY, $J$ Dent Res 44:350-354, 1965). Moreover, overall sexual dimorphism in root length, calculated as $100(\mathrm{M} / \mathrm{F}-1.00)$, proves to be of the order of $6 \%$ for the sample investigated, larger than percent sexual dimorphism for the corresponding crown dimensions.

Reviewing factors that might affect the correlations, it is clear that variations in subject positioning and differences in jaw form would tend to attenuate correlations. Conversely, differences in jaw size and intraindividual similarities in root-angulation could augment intraindividual root length correlations. However, for the tube-to-film and tube-to-median plane-distances involved, and the range of enlargement ratios then possible, these latter variables would not account for more than a very small fraction of observed root-length variance and, therefore, for more than slight inflation of rootlength correlations. Accordingly, the intraindividual root-length correlations presented here provide evidence for a useful third dimension in odontometric analyses both of normal individuals and those with congenital malformation syndromes and karyotypic abnormalities.

TABLE

ROOT-LENGTH INTERCORRELATIONS FOR 122 INDIVIDUALS*

\begin{tabular}{lccccc}
$\begin{array}{c}\text { Mandibular } \\
\text { Toothi }\end{array}$ & $\mathrm{C}$ & $\mathrm{P}_{1}$ & $\mathrm{P}_{2}$ & $\mathrm{M}_{1}$ & $\mathrm{M}_{2}$ \\
\hline $\mathrm{C}$ & - & 0.65 & 0.44 & 0.44 & 0.35 \\
$\mathrm{P}_{1}$ & - & - & 0.67 & 0.51 & 0.48 \\
$\mathrm{P}_{2}$ & - & - & - & 0.56 & 0.57 \\
$\mathrm{M}_{1}$ & - & - & - & - & 0.62 \\
\hline
\end{tabular}

* Pooled $r$ from sex-specific values of $r$. Root length measurements cemento-enamel junction to apex, a veraged for multiple-rooted teeth. 